\title{
The Riemann-Cartan space in the O-theory
}

\author{
V. Yu. Dorofeev* \\ Friedmann Laboratory for Theoretical Physics
}

\begin{abstract}
Nonrelativistic equation of particle with a spin for the Lagrangian on a nonassociative algebra is obtained. It is shown that in this model arises Riemann-Cartan space. In the case of central symmetry in addition to the pseudo-curvature appears torsion as pseudovector that interacts with the spin of the particle. An estimation of the influence of torsion on the strength of gravitational attraction in the central gravitational field is given.
\end{abstract}

\section{Introduction}

In the paper [1] the author proposes a generalization of the Lagrangian theory of Weinberg-Salam on the nonassociative algebra. In the paper [2] the author considered some physical consequences of the nonassociative Lagrangian. In particular, it was found that the proposed model is consistent with geometric solutions of general relativity types of Schwardzchild and Friedmann metric, and Einstein's equations of the form

$$
T_{\mu \nu}=\lambda R_{\mu \nu}
$$

Octonions as an algebraic structure for the construction of physical theories are used by a number of authors ([3], [4], etc.). In order to identity the theory, which is based on the Lagrangian, developed in [1], the name of O-theory is proposed.

This work is a direct continuation of the research started in [1] and [2].

\section{Concepts of the O-theory}

Recall some fundamental aspects of O-theory [1].

Compensating fields $\mathbf{A}_{\mu}(x), x \in M_{4}$ in the Minkowski space $M_{4}$ of nonassociative Lagrangian $\mathfrak{L}_{o}$ have the form:

$$
\mathbf{A}_{\mu}(x)=A_{\mu}^{i}(x) \Sigma^{i}, i=0,1,2, \ldots, 7
$$

*Dep. of Math., SPb SUEF, Sadovaya 21, 191023, St.Petersburg, Russia, E-mail: friedlab@mail.ru 
where $\Sigma^{i}$ are generators of the algebra $\mathcal{O}$ and $A_{\mu}^{i}(x)$ are vector fields.

Nonassociative algebra is represented by the matrices $(i=1,2,3)$

$$
\begin{array}{rlr}
\Sigma^{0}=\left(\begin{array}{cc}
\sigma^{0} & 0 \\
0 & \sigma^{0}
\end{array}\right) & \Sigma^{i}=\left(\begin{array}{cc}
0 & -i \sigma^{i} \\
i \sigma^{i} & 0
\end{array}\right) \\
\Sigma^{4}=\left(\begin{array}{cc}
-\sigma^{0} & 0 \\
0 & \sigma^{0}
\end{array}\right) & \Sigma^{4+i}=\left(\begin{array}{cc}
0 & -\sigma^{i} \\
-\sigma^{i} & 0
\end{array}\right)
\end{array}
$$

where $\sigma^{0}$ is an identity matrix and $\sigma^{i}, i=1,2,3$ are Pauli matrices:

$$
\sigma^{1}=\left(\begin{array}{ll}
0 & 1 \\
1 & 0
\end{array}\right), \quad \sigma^{2}=\left(\begin{array}{cc}
0 & -i \\
i & 0
\end{array}\right), \quad \sigma^{3}=\left(\begin{array}{cc}
1 & 0 \\
0 & -1
\end{array}\right), \quad \sigma^{0}=\left(\begin{array}{ll}
1 & 0 \\
0 & 1
\end{array}\right)
$$

with a special law of multiplication

$$
\begin{gathered}
\mathbf{o} * \mathbf{o}^{\prime}=\left(\begin{array}{cc}
\lambda I & A \\
B & \xi I
\end{array}\right) *\left(\begin{array}{cc}
\lambda^{\prime} I & A^{\prime} \\
B^{\prime} & \xi^{\prime} I
\end{array}\right)= \\
=\left(\begin{array}{ll}
\left(\lambda \lambda^{\prime}+\frac{1}{2} \operatorname{tr}\left(A B^{\prime}\right)\right) I & \lambda A^{\prime}+\xi^{\prime} A+\frac{i}{2}\left[B, B^{\prime}\right] \\
\lambda^{\prime} B+\xi B^{\prime}-\frac{i}{2}\left[A, A^{\prime}\right] & \left(\xi \xi^{\prime}+\frac{1}{2} \operatorname{tr}\left(B A^{\prime}\right)\right) I
\end{array}\right)
\end{gathered}
$$

In the last formula we introduced the matrix $A, A^{\prime}, B, B$ of dimension $(2 \times 2)$, identity matrix $I=\sigma^{0}$ and complex numbers $\lambda, \xi, \lambda^{\prime}, \xi^{\prime}$.

States space is based on the expanded space $\mathfrak{O}$ of the algebra $\mathcal{O}_{\mathbb{C}}$. The basis of this space is formed by matrixes

$$
f^{a}=\Sigma^{a}, \quad a=0,1, \ldots, 7, f^{8}=\left(\begin{array}{cc}
0 & I \\
I & 0
\end{array}\right), \quad f^{9}=\left(\begin{array}{cc}
0 & i I \\
-i I & 0
\end{array}\right)
$$

keeping product from (4).

By means of multiplication (4) we introduce convolution:

$$
\begin{aligned}
\left(\overline{\mathbf{o}}_{1}, \overline{\mathbf{o}}_{2}\right) & =\frac{1}{2} \operatorname{tr}\left(\overline{\mathbf{o}}_{1}^{+} * \overline{\mathbf{o}}_{2}\right)=\frac{1}{2} \operatorname{tr}\left(\left(\begin{array}{cc}
\lambda_{1}^{*} I & B_{1}^{+} \\
A_{1}^{+} & \xi_{1}^{*} I
\end{array}\right) *\left(\begin{array}{cc}
\lambda_{2} I & A_{2} \\
B_{2} & \xi_{2} I
\end{array}\right)\right) \\
& \left.=\lambda_{1}^{*} \lambda_{2}+\xi_{1}^{*} \xi_{2}+\frac{1}{2} \operatorname{tr}\left(B_{1}^{+} B_{2}\right)\right)+\frac{1}{2} \operatorname{tr}\left(A_{1}^{+} A_{2}\right)
\end{aligned}
$$

where $\mathfrak{O} \times \mathfrak{O}$ maps to $\mathbb{C}$.

\section{Nonassociative Lagrangian}

In the work [1] the proposed Lagrangian, generalising Weinberg-Salam Lagrangian (Standart Theory $=\mathrm{ST}$ ) on the nonassociative algebra, has a following form $(a, b=0,1, \ldots, 7, k=1,2, \ldots, 7)$ :

$$
\begin{aligned}
\mathfrak{L}_{\text {oct. }}= & \mathfrak{L}_{f}+\left(\partial_{\mu} \stackrel{*}{\Psi}_{\varphi}-\frac{i}{2} q^{a} A_{\mu}^{a} \stackrel{*}{\Psi}_{\varphi} * \Sigma^{a}\right) *\left(\partial^{\mu} \Psi_{\varphi}+\frac{i}{2} q^{b} A^{\mu(b)} \Sigma^{b} * \Psi_{\varphi}\right) \\
& +\frac{i}{2} \bar{L} * \gamma_{\mu}\left(\vec{\partial}^{\mu} L+\frac{i}{2} c_{L} q^{k} A^{\mu(k)} \Sigma^{k} * L+\frac{i}{2} c_{L_{0}} q^{0} A^{\mu(0)} L\right)
\end{aligned}
$$




$$
\begin{gathered}
-\frac{i}{2} \bar{L} * \gamma_{\mu}\left(\overleftarrow{\partial}^{\mu} L-\frac{i}{2} c_{L} q^{k} A^{\mu(k)} \Sigma^{k} * L-\frac{i}{2} c_{L_{0}} q^{0} A^{\mu(0)} L\right) \\
+\frac{i}{2} \bar{R} \gamma_{\mu}\left(\vec{\partial}^{\mu} R+i q^{0} A^{\mu 0} R\right)-\frac{i}{2} \bar{R} \gamma_{\mu}\left(\overleftarrow{\partial}^{\mu} R-i q^{0} A^{\mu 0} R\right) \\
\left.\quad-\tilde{h} \bar{L} * \Psi_{\varphi} R-\tilde{h} \bar{R} \stackrel{*}{\Psi}_{\varphi} * L\right)+m^{2}\left\|\Psi_{\varphi}\right\|^{2}-\frac{f}{4}\left\|\Psi_{\varphi}\right\|^{4}
\end{gathered}
$$

Denote Lagrangian of free fields $\mathfrak{L}_{f}$

$$
\begin{gathered}
\mathfrak{L}_{f}=-\frac{1}{4} F_{\mu \nu}^{0} F^{\mu \nu(0)}-\frac{1}{4} \operatorname{tr}\left(F_{\mu \nu}^{k} * F^{\mu \nu(k)}\right) \\
+\frac{1}{16} f^{i j k l}\left(A_{\mu}^{i} A_{\nu}^{j}-A_{\nu}^{j} A_{\mu}^{i}\right)\left(A^{\mu(k)} A^{\nu(l)}-A^{\nu(k)} A^{\mu(l)}\right) \\
f^{i j k l}=q^{i j} q^{k l} \operatorname{tr}\left(\Sigma^{i} * \Sigma^{j} * \Sigma^{k} * \Sigma^{l}\right) \\
F_{\mu \nu}^{0}=\partial_{\mu} A_{\nu}^{0}-\partial_{\nu} A_{\mu}^{0}, \quad F_{\mu \nu}^{k}=\partial_{\mu} A_{\nu}^{k}-\partial_{\nu} A_{\mu}^{k}-\varepsilon^{i j k} q^{i j}\left(A_{\mu}^{i} A_{\nu}^{j}-A_{\nu}^{i} A_{\mu}^{j}\right)
\end{gathered}
$$

The values $f^{i j k l}, i, j, k, l=1, \ldots, 7$ reflect nonassociative character of $\mathfrak{L}_{f}$. Introduce the left and the right components of spinors

$$
\frac{1}{2}\left(1+\gamma^{5}\right) \Psi=L, \quad \frac{1}{2}\left(1-\gamma^{5}\right) \Psi=R, \quad \gamma^{5}=i \gamma^{0} \gamma^{1} \gamma^{2} \gamma^{3}
$$

$\Psi, \Psi_{\varphi}$ are vectors of states from $\mathfrak{O}$ which elements are bispinors; $q^{a}, c_{L}, c_{L_{0}}$ are some numbers caused by normalization; $\gamma^{\mu}$ are Dirac's matrices $(\mu=$ $0,1,2,3 ; i=1,2,3)$ :

$$
\gamma^{0}=\left(\begin{array}{cc}
I & 0 \\
0 & -I
\end{array}\right), \quad \gamma^{i}=\left(\begin{array}{cc}
0 & \sigma^{i} \\
-\sigma^{i} & 0
\end{array}\right), \quad \gamma^{5}=\left(\begin{array}{cc}
0 & I \\
I & 0
\end{array}\right)
$$

where different-case Greek indexes specify summation by the metric tensor of Minkowski space $\eta_{\mu \nu}$ with the signature $(1,-1,-1,-1)$, and the indexes of the same case specify the simple summation.

Furthermore, in [1] in the basic vacuum state

$$
\Psi_{\varphi}=\Psi_{0}=\frac{m}{\sqrt{2 f}}\left(\begin{array}{cc}
0 & i \sigma^{3} \\
0 & I
\end{array}\right)
$$

the general expression of nonassociative Lagrangian for lepton sector is derived

$$
\begin{aligned}
& \mathfrak{L}_{o}=\mathfrak{L}_{f}+ \frac{q^{(k) 2} m^{2}}{2 f} A_{\mu}^{k} A^{\mu(k)}+\frac{o^{i j} m^{2}}{2 f} A_{\mu}^{i} A^{\mu(j)}+\frac{g^{(1) 2} m^{2}}{2 f} B_{\mu} B^{\mu}-\frac{g g^{(1)} m^{2}}{f} A_{\mu}^{3} B^{\mu} \\
&+ \frac{g^{(1)}}{2} \bar{\nu}_{L} \gamma_{\mu} B^{\mu} \nu_{L}+\frac{g^{(1)}}{2} \bar{e}_{L} \gamma_{\mu} B^{\mu} e_{L}+\frac{g}{2} \bar{e}_{L} \gamma_{\mu} A^{\mu 3} e_{L}-\frac{g}{2} \bar{\nu}_{L} \gamma_{\mu} A^{\mu 3} \nu_{L} \\
&-\frac{g}{2} \bar{\nu}_{L} \gamma_{\mu} e_{L}\left(A^{\mu 1}-i A^{\mu 2}\right)-\frac{g}{2} \bar{e}_{L} \gamma_{\mu} \nu_{L}\left(A^{\mu 1}+i A^{\mu 2}\right) \\
&+\frac{i}{2}\left(\bar{e}_{L} \gamma_{\mu} \partial^{\mu} e_{L}-\partial^{\mu} \bar{e}_{L} \gamma_{\mu} e_{L}\right)+\frac{i}{2}\left(\bar{\nu}_{L} \gamma_{\mu} \partial^{\mu} \nu_{L}-\partial^{\mu} \bar{\nu}_{L} \gamma_{\mu} \nu_{L}\right)+\frac{m^{4}}{f}
\end{aligned}
$$




$$
\begin{gathered}
+\frac{i}{2}\left(\bar{e}_{R} \gamma_{\mu} \partial^{\mu} e_{R}-\partial^{\mu} \bar{e}_{R} \gamma_{\mu} e_{R}\right)+g^{(1)} \bar{e}_{R} \gamma_{\mu} B^{\mu} e_{R}-\frac{\sqrt{2} h m}{\sqrt{f}}\left(\bar{e}_{L} e_{R}+\bar{e}_{R} e_{L}\right) \\
-q^{4} A^{\mu(4)}\left(\kappa_{1} \bar{\nu}_{L} \gamma_{\mu} \nu_{L}-\kappa_{2} \bar{e}_{L} \gamma_{\mu} e_{L}\right)-\frac{3}{2} q^{6} A^{\mu(6)} \bar{e}_{L} \gamma_{\mu} e_{L} \\
-\frac{5}{4}\left(q^{6} A^{\mu(6)}+i q^{5} A^{\mu(5)}\right) \bar{\nu}_{L} \gamma_{\mu} e_{L}-\frac{5}{4}\left(q^{6} A^{\mu(6)}-i q^{5} A^{\mu(5)}\right) \bar{e}_{L} \gamma_{\mu} \nu_{L}
\end{gathered}
$$

with the following denotes $i c_{2}^{i j} A^{i j} q^{i} q^{j}=o^{i j}$.

In [2] it is shown that in case of a certain relation between the senior fields $A_{\mu}^{i}, i=4,5,6,7$ and charge constants $q^{i j}, i, j=4,5,6,7$ it follows from Lagrangian (12) that a gravitational field consists of two oppositely charged massless vector bosons $D_{\mu}$ and $D_{\mu}^{*}$. Thus the Dirac equation for lepton sector, with the exception of electromagnetic interaction, looks like:

$$
\left(i \gamma^{\mu}\left(\partial_{\mu}-i q_{D} D_{L}^{\mu}\right)-m_{e}\right) e(x)=0
$$

where

$$
q_{D} D^{\mu}=-\frac{3}{2} q^{6} A^{\mu(6)}-i \frac{3}{2} q^{5} A^{\mu(5)}
$$

and $D_{L}^{\mu}=D^{\mu} \frac{1}{2}\left(1+\gamma^{5}\right)$.

\section{The Dirac equation in Riemannian space}

Let $M$ be a pseudoriemannian manifold where it is possible to introduce coordinates $\left(x^{0}, x^{1}, x^{2}, x^{3}\right)$ and metric

$$
d s^{2}=g_{\mu \nu} d x^{\mu} d x^{\nu}
$$

Let $\delta A^{\mu}=\Gamma_{\nu \lambda}^{\mu} A^{\nu} d x^{\lambda}[6]$, where $\Gamma_{\nu \lambda}^{\mu}$

$$
\Gamma_{\nu \lambda}^{\mu}=\frac{1}{2} g^{\mu \kappa}\left(g_{\mu \kappa, \nu}+g_{\nu \kappa, \lambda}-g_{\lambda \nu, \kappa}\right)
$$

Then for a covariant derivative

$$
A_{; \nu}^{\mu}=A_{, \nu}^{\mu}+\Gamma_{\nu \lambda}^{\mu} A^{\lambda}
$$

the transformation to the new coordinates is equivalent to the transformation of a vector in Riemannian space.

Christoffel symbols don't form a tensor. The combination of Christoffel symbols is a Riemann tensor which we define in accordance to [6]

$$
R_{\mu \nu \lambda}^{\tau}=\Gamma_{\mu \lambda, \nu}^{\tau}-\Gamma_{\mu \nu, \lambda}^{\tau}+\Gamma_{\sigma \nu}^{\tau} \Gamma_{\mu \lambda}^{\sigma}-\Gamma_{\sigma \lambda}^{\tau} \Gamma_{\mu \nu}^{\sigma} .
$$

satisfies to the tensor law of transformation.

The quadratic form (15) in a vicinity of some point can be reduced to a diagonal form

$$
d s^{2}=H^{(0) 2} d x^{(0) 2}-H^{(1) 2} d x^{(1) 2}-H^{(2) 2} d x^{(2) 2}-H^{(3) 2} d x^{(3) 2}
$$


By associating the diagonal coordinates with the coordinates of the physical space-time, we believe that in some coordinates the metric is given by the diagonal form of Minkowski space $M_{4}$ :

$$
d s^{2}=c^{2} d t^{2}-d x^{2}-d y^{2}-d z^{2}=\eta_{a b} d x^{a} d x^{b}
$$

(In this section we will distinguish Greek and Latin indices, assuming that the Greek indices refer to the pseudoriemannian space, while Latin refer to Euclidean one.)

Suppose that at each point of a pseudoriemannian space $M$ there is defined a stratification, which is the tangent Minkowski space $M_{4}$ with the metric (20).

Thus the pseudoriemannian coordinates of the physical space-time and the coordinates of the tangent space are given by the formulas:

$$
H^{(0)} d x^{(0)}=c d t, H^{(1)} d x^{(1)}=d x, H^{(2)} d x^{(2)}=d y, H^{(3)} d x^{(3)}=d z
$$

On the other hand, in each point of pseudoriemannian space $M$ there can be introduced tetrads $h_{\mu}^{a}$, connecting the metric of pseudoriemannian space to the metric of Minkowski space:

$$
h_{\mu}^{b} h_{\nu}^{\mu}=\delta_{a}^{b}, \quad h^{\mu(a)} h_{a}^{\nu}=g^{\mu \nu}
$$

Any vector pseudoriemannian spaces $A_{\mu}$ can be presented componentwise in space Minkovsky $M_{4}$ by means of tetrads $h_{\mu}^{a}$ as $A^{a}=A^{\mu} h_{\mu}^{a}$, therefore (17)

$$
\delta A^{\mu}=\delta\left(A^{a} h_{a}^{\mu}\right)=\delta A^{a} h_{a}^{\mu}+A^{a} \delta h_{a}^{\mu}=\delta A^{a} h_{a}^{\mu}+A^{a} h_{a, \nu}^{\mu} \delta x^{\nu}=\Gamma_{\nu \lambda}^{\mu} A^{\nu} \delta x^{\lambda}
$$

With the assumption $h_{\mu}^{b} h_{a, \nu}^{\mu}+h_{\mu, \nu}^{b} h_{a}^{\mu}=0$, find

$$
\delta A^{b}=\gamma_{a c}^{b} A^{a} \delta x^{c}, \quad \gamma_{a c}^{b}=h_{\mu ; \nu}^{b} h_{a}^{\mu} h_{c}^{\nu}
$$

where $\gamma_{a c}^{b}$ are the factors of rotation of Ricci.

In the work of Fock-Ivanenko [5] it is shown that the free Dirac equation

$$
\left(i \gamma^{a} \partial_{a}-m\right) e(x)=0
$$

rewritten in $M_{4}$ as in tangent layer of a pseudoriemannian manifold looks like

$$
\left(i \gamma^{a}\left(\partial_{a}-\Gamma_{a}\right)-m\right) e(x)=0
$$

where

$$
\Gamma_{a}=-\frac{1}{2} \gamma_{a b c} \sigma_{b c}, \quad \sigma^{a b}=\frac{1}{4}\left[\gamma^{a}, \gamma^{b}\right], \quad a, b=0,1,2,3
$$

Dirac matrices $\gamma^{a}, a=0, \ldots, 3$ satisfy to the following multiplication law with matrix $\sigma^{a b}$ :

$$
\gamma^{a} \sigma^{b c}=\frac{1}{4} \gamma^{a}\left[\gamma^{b}, \gamma^{c}\right]=\frac{1}{2} \eta^{a b} \gamma^{c}-\frac{1}{2} \eta^{a c} \gamma^{b}-\frac{i}{2} \varepsilon^{d a b c} \gamma^{5} \gamma_{d}
$$


Hence

$$
-\gamma^{a} \Gamma_{a}=\frac{1}{4} h_{a}^{\mu} h_{b}^{\nu} h_{(c) \nu ; \mu}\left(\frac{1}{2} \eta^{a b} \gamma^{c}-\frac{1}{2} \eta^{a c} \gamma^{b}-\frac{i}{2} \varepsilon^{d a b c} \gamma^{5} \gamma_{d}\right)
$$

Let the metric of pseudoriemannian space have the form (19) therefore for nonequal values of $a, b, c$ we have

$$
\Gamma_{\mu \nu}^{\lambda} h_{c \lambda} h_{b}^{\mu} h_{c}^{\nu}=\frac{1}{2} g^{\mu \kappa}\left(g_{\mu \kappa, \nu}+g_{\nu \kappa, \lambda}-g_{\lambda \nu, \kappa}\right) h_{c \lambda} h_{b}^{\mu} h_{c}^{\nu}=0
$$

hence

$$
\begin{gathered}
-\gamma^{a} \Gamma_{a}=\frac{1}{4} h_{a}^{\mu} h^{\nu(a)} h_{(c) \nu ; \mu} \gamma^{c}-\frac{1}{4} h^{\mu(a)} h_{b}^{\nu} h_{(a) \nu ; \mu} \gamma^{b} \\
=\frac{1}{4} h_{c ; \mu}^{\mu} \gamma^{c}+\frac{1}{4} h^{\mu(a)} h_{b ; \mu}^{\nu} h_{(a) \nu} \gamma^{b}=\frac{1}{2} h_{c ; \mu}^{\mu} \gamma^{c}
\end{gathered}
$$

and the Dirac equation forms [7]:

$$
\left(\gamma^{a}\left(H^{a}\right)^{-1}\left(\partial_{a}-i \Phi_{a}+\frac{1}{2} \partial_{a}\left(\ln \frac{\sqrt{-g}}{H^{a}}\right)\right)-m\right) \psi=0
$$

In a spherically symmetric coordinate system relatively to the center of the Earth, space-time metric can be chosen in the following way

$$
d s^{2}=\left(1-\frac{r_{g}}{r}\right) d t^{2}-\frac{d r^{2}}{1-\frac{r_{g}}{r}}-r^{2}\left(\sin ^{2} \theta d \varphi^{2}+d \theta^{2}\right)
$$

where $r_{g}=2 k M / R$ is Schwarzchild radius.

In a gravitational field the Dirac equation (26) relatively to the metric of Schwarzchild (33) becomes [9]

$$
\left(\gamma^{0} \frac{1}{f} \partial_{t}+\gamma^{r} f \partial_{r}-\gamma^{r} \frac{\vec{\Sigma} \cdot \hat{\vec{L}}}{r}-i m\right) e=-\gamma^{r}\left(\frac{1}{2} f_{, r}+\frac{1}{r}(f-1)\right) e,
$$

where $\vec{L}=\vec{r} \times \vec{p}$ is angular momentum operator, $f^{2}=1-\frac{r_{g}}{r}$,

$$
\gamma^{r}=\gamma^{1} \sin \theta \cos \varphi+\gamma^{2} \sin \theta \sin \varphi+\gamma^{3} \cos \theta, \quad \vec{\Sigma}=\left(\begin{array}{cc}
\vec{\sigma} & 0 \\
0 & \vec{\sigma}
\end{array}\right) .
$$

By substituting $f$ in (34) we find that in the first approximation

$$
\frac{1}{2} f_{, r}+\frac{1}{r}(f-1)=-\frac{r_{g}}{4 r^{2}}
$$

From (35) figure out that

$$
q^{5} A_{\mu}^{5}=\left(0,-\frac{r_{g}}{3 r^{2}}, 0,0\right)
$$

On the other hand, the equation of motion for the charged vector boson $D_{\mu}$ is a wave equation for massless particle [2]. Hence drawing an analogy with an 
electromagnetic field for which stationary real component of $1 / r$, caused by the energy conservation law [8], is selected, we also will be limited by the stationary component and we assume that

$$
q^{5} A_{\mu}^{5}=q^{6} A_{\mu}^{6}
$$

or

$$
\begin{aligned}
q^{5} A_{r}^{5}=\frac{2 k M \hbar}{3 c^{3} r^{2}}=\frac{2 g \hbar}{3 c} & =9.8 \cdot 10^{2} \mathrm{~cm} / \mathrm{c}^{2} \cdot 10^{-27} \mathrm{erg} \cdot \mathrm{c} \cdot 0.22 \cdot 10^{-10} \mathrm{c} / \mathrm{cm} \\
& =0.2 \cdot 10^{-34} \mathrm{erg} \approx 10^{-23} \mathrm{eV}
\end{aligned}
$$

\section{Dirac equation in the Riemann-Cartan space}

Covariant derivative of (17) will be a vector and if the Christoffel symbols contain an asymmetric part of the lowercase indices. In this case, pseudoriemannspace is generalized to Riemann-Cartan or $U^{4}$ space in which the torsion is equal to the antisymmetric part of the object

$$
Q_{\mu \nu}^{\lambda}=\frac{1}{2}\left(\Gamma_{\mu \nu}^{\lambda}-\Gamma_{\nu \mu}^{\lambda}\right)
$$

regardless to the pseudo-metric $g_{\mu \nu}$.

Torsion tensor has 24 independent components and can be decomposed into a sum of three irreducible parts

$$
Q_{\mu \nu}^{\lambda}=\tilde{Q}_{\mu \nu}^{\lambda}+\frac{1}{3}\left(\delta_{\mu}^{\lambda} Q_{\nu}-\delta_{\nu}^{\lambda} Q_{\mu}\right)+\varepsilon_{\sigma \mu \nu \alpha} g^{\sigma \lambda} \breve{Q}^{\alpha}
$$

where $\tilde{Q}_{\mu \nu}^{\lambda}$ is traceless part of the torsion tensor, $Q_{\mu}$ is trace of the torsion tensor

$$
Q_{\mu}=Q_{\mu \lambda}^{\lambda}
$$

and $\breve{Q}^{\alpha}$ is pseudotrace of the torsion tensor

$$
\breve{Q}_{\alpha}=\frac{1}{3 !} \varepsilon_{\alpha \mu \nu \sigma} Q^{\mu \nu \sigma}
$$

(lifting and lowering indices can be carried out by the metric tensor $g^{\mu \nu}$ ).

The part of the object of connectivity, which is symmetric by the lower-case indexes $\Gamma_{\mu \nu}^{\lambda}$, is not generally aligned to the metrics. However further we will

limit our consideration by the case when its symmetric part is defined by a metric tensor.

$$
\dot{\Gamma}_{\mu \nu}^{\lambda}=\frac{1}{2}\left(\Gamma_{\mu \nu}^{\lambda}+\Gamma_{\nu \mu}^{\lambda}\right)=\frac{1}{2} g^{\lambda \sigma}\left(g_{\nu \sigma, \mu}+g_{\mu \sigma, \nu}-g_{\mu \nu, \sigma}\right)
$$

hence

$$
\Gamma_{\mu \nu}^{\lambda}=\dot{\Gamma}_{\mu \nu}^{\lambda}+Q_{\mu \nu}^{\lambda}
$$


Then the Ricci coefficeint of rotation (24) is generalized to

$$
\begin{gathered}
\gamma_{a c}^{b}=\left(h_{\mu, \nu}^{b}+\Gamma_{\mu \nu}^{\lambda} h_{\lambda}^{b}\right) h_{a}^{\mu} h_{c}^{\nu}=\left(h_{\mu, \nu}^{b}+\dot{\Gamma}_{\mu \nu}^{\lambda} h_{\lambda}^{b}+Q_{\mu \nu}^{\lambda} h_{\lambda}^{b}\right) h_{a}^{\mu} h_{c}^{\nu}= \\
=\left(h_{\mu ; \nu}^{b}+Q_{\mu \nu}^{\lambda} h_{\lambda}^{b}\right) h_{a}^{\mu} h_{c}^{\nu}
\end{gathered}
$$

In some map let's reduce the quadratic form to a diagonal form $M_{4}$ and we will consider the case with traceless and vector parts of the tensor of torsion are equal to zero. Then for distinct values $a, b, c$ in contrast to (30) we will get

$$
\Gamma_{\mu \nu}^{\lambda} h_{c \lambda} h_{b}^{\mu} h_{c}^{\nu}=Q_{\mu \nu}^{\lambda} h_{c \lambda} h_{b}^{\mu} h_{c}^{\nu} \neq 0
$$

and

$$
-\gamma^{a} \Gamma_{a}=\frac{1}{2} h_{c \tilde{\mu}}^{\mu} \gamma^{c}+i \gamma^{5} \gamma_{d}\left(-\frac{1}{8} \varepsilon^{d a b c} h_{a}^{\mu} h_{b}^{\nu} h_{(c) \lambda} Q_{\mu \nu}^{\lambda}\right)
$$

and the Dirac equation takes the form:

$$
\left(\gamma^{a}\left(H^{a}\right)^{-1}\left(\partial_{a}-i \Phi_{a}+\frac{1}{2} \partial_{a}\left(\ln \frac{\sqrt{-g}}{H^{a}}\right)+i \gamma^{5} A_{a}\right)-m\right) \psi=0
$$

where $A^{d}=-\frac{1}{8} \varepsilon^{d a b c} h_{a}^{\mu} h_{b}^{\nu} h_{(c) \lambda} Q_{\mu \nu}^{\lambda}$.

On the other hand, formerly the Dirac equation on the nonassociative algebra was obtained in the form (13). Let's substitute (14) in (13):

$$
\left(\gamma^{\mu}\left(\partial_{\mu}+i \frac{3}{4} q^{6} A_{\mu}^{6}+i \frac{3}{4} q^{6} A_{\mu}^{6} \gamma^{5}+\frac{3}{4} q^{5} A_{\mu}^{5}+\frac{3}{4} q^{5} A_{\mu}^{5} \gamma^{5}\right)+i m\right) e(x)=0
$$

In the long derivative the equation has two vectors and two pseudovectors. Each of these members we consider separately. For this purpose introduce an index $\epsilon_{g_{i}}=0,1$, then the long derivative of the Dirac equation (49) becomes

$$
\partial_{\mu}+i \epsilon_{g_{1}} \frac{3}{4} q^{6} A_{\mu}^{6}+i \epsilon_{g_{2}} \frac{3}{4} q^{6} A_{\mu}^{6} \gamma^{5}+\epsilon_{g_{3}} \frac{3}{4} q^{5} A_{\mu}^{5}+\epsilon_{g_{4}} \frac{3}{4} q^{5} A_{\mu}^{5} \gamma^{5}
$$

Assume $\epsilon_{g_{1}}=1, \epsilon_{g_{i}}=0, i=2,3,4$. Then we have an analogy to lengthening of the derivative caused by the electromagnetic vector-potential

$$
\left(\gamma^{\mu}\left(\partial_{\mu}+i \frac{3}{4} q^{6} A_{\mu}^{6}\right)+i m\right) e(x)=0
$$

Therefore we can assume that this term is caused by the interaction of the electron with the charged pair of $D+D^{*}$-bosons (as a whole it is electrically neutral). However since it is several orders smaller than the conventional electromagnetic interaction of the electron with surrounding charges of [2], in general considered as electrically neutral, that small-order member is to be neglected.

Assume $\epsilon_{g_{3}}=1, \epsilon_{g_{i}}=0, i=1,2,4$. Then we have an analogy to lengthening of the derivative as a tetrad representation of the Dirac equation, rewritten in a Riemannian space in a diagonal metric (48)

$$
\left(\gamma^{\mu}\left(\partial_{\mu}+\frac{3}{4} q^{5} A_{\mu}^{5}\right)+i m\right) e(x)=0
$$


Assume $\epsilon_{g_{2}}=1, \epsilon_{g_{i}}=0, i=1,3,4$. Then we have an analogy to lengthening of the derivative as a tetrad representation of the Dirac equation, rewritten in a Riemannian-Cartan space

$$
\left(i \gamma^{\mu}\left(\partial_{\mu}+i \gamma^{5} \frac{3}{4} q^{6} A_{\mu}^{6}\right)-m\right) e(x)=0
$$

Finally, if $\epsilon_{g_{4}}=1, \epsilon_{g_{i}}=0, i=1,2,3$, then in fact it is the manifestation of the axial properties of the electromagnetic current (similarly to ST), which is neglected everywhere below.

\section{Nonrelativistic electron in the space of Riemann-Cartan}

Consider the equation of the electron in the form (53), assuming (37). Let

$$
\Psi=e^{-i \varepsilon t / h}\left(\begin{array}{c}
\varphi(r) \\
\xi(r)
\end{array}\right)
$$

then, denoting $T^{c}=\left(0, \frac{r_{g}}{4 r^{2}}, 0,0\right)$, obtain

$$
\left\{\begin{array}{l}
\varepsilon \varphi+c \vec{\sigma} \vec{p} \xi-m c^{2} \varphi=-\hbar c \sigma^{r} T_{r}^{c} \varphi \\
\varepsilon \xi+c \vec{\sigma} \vec{p} \varphi+m c^{2} \xi=-\hbar c \sigma^{r} T_{r}^{c} \xi
\end{array}\right.
$$

or, introducing $E^{\prime}=\varepsilon-m c^{2}$, have

$$
\left\{\begin{array}{c}
\left(E^{\prime}+\hbar c \vec{\sigma} \vec{T}^{c}\right) \varphi=-c \vec{\sigma} \vec{p} \xi \\
\text { displaystyle }\left(E^{\prime}+2 m c^{2}+\hbar c \vec{\sigma} \vec{T}^{c}\right) \xi=-c \vec{\sigma} \vec{p} \varphi
\end{array}\right.
$$

If $\left|E^{\prime}+T_{r}^{c}\right|<<2 m c^{2}$ then

$$
\xi=-\left(E^{\prime}+2 m c^{2}+\hbar c \vec{\sigma} \vec{T}^{c}\right)^{-1} c \vec{\sigma} \vec{p} \varphi \approx-\frac{1}{2 m c} \vec{\sigma} \vec{p} \varphi
$$

Substitute (57) in (56). Then for the component $\varphi$ of the bispinor $\Psi$ we get:

$$
E^{\prime} \varphi \approx\left(\frac{1}{2 m}(\vec{\sigma} \vec{p})^{2}-\hbar c \vec{\sigma} \vec{T}^{c}\right) \varphi
$$

From the quantum equation (58) it follows that nonrelativistic particle, with the spin $\vec{\sigma}$, in the field of the Earth has an energy

$$
E=\frac{\vec{p}^{2}}{2 m}+V_{g}+V_{c}
$$

where $V_{g}$ is gravitational energy of the particle and $V_{c}$ is potential energy of the torsion of Cartan

$$
V_{c}=-\hbar c \vec{\sigma} \vec{T}^{c}=-\frac{\hbar r_{g} c}{4 r^{3}}(\vec{\sigma} \vec{r})
$$


Thus, in the gravitational field of a massive spherical body the torsion manifests itself as a shift of spinor particle energy on the value of

$$
\Delta E_{c}=\frac{\hbar r_{g} c}{4 r^{3}}(\vec{\sigma} \vec{r})=\frac{k M \hbar}{2 c r^{3}}(\vec{\sigma} \vec{r})
$$

were $k=6.67 \cdot 10^{-8} \mathrm{~cm}^{3} \mathrm{~g}^{-1} \mathrm{c}^{-2}$ - gravitational constant, $M$ - mass of Earth, $r_{g}=0.9 \mathrm{~cm}$

$$
\begin{aligned}
\Delta E=\frac{k M \hbar}{2 c r^{2}}=\frac{g \hbar}{2 c} & =9.8 \cdot 10^{2} \mathrm{~cm} / \mathrm{c}^{2} \cdot 10^{-27} \mathrm{erg} \cdot \mathrm{c} \cdot 0.17 \cdot 10^{-10} \mathrm{c} / \mathrm{cm} \\
& =0.17 \cdot 10^{-34} \mathrm{erg} \approx 10^{-23} \mathrm{eV}
\end{aligned}
$$

This is sufficiently small value. On the other hand, consider $1 \mathrm{~cm}^{3}$ of the magnetized metal, in which all the spins, causing its magnetic properties, are oriented in the same direction. Since the number of spin particles in the volume is equal to $N=10^{-23}$, then the energy change due to torsion is

$$
\Delta E_{c}=N \cdot \Delta E=10^{23} \cdot 10^{-23} \mathrm{eV}=1 \mathrm{eV}
$$

Now it is more significant. The effect will be even more meaningful for the ferromagnet, in which a single atom will have several same-oriented electrons.

Another estimation of the impact of torsion to the satellite is interesting. Let the satellite be an oriented permanent magnet. Its energy is

$$
E=\frac{m v^{2}}{2}-k \frac{M m}{r}-\frac{N \hbar r_{g}(\hat{\vec{\sigma}} \cdot \vec{r})}{4 c r^{3}}
$$

where $N$ is a number of the spins.

Suppose there is a rotation around a circular orbit with a total energy equal to zero. Then $v=\Omega r$ and

$$
\frac{m}{2} \Omega^{2} r^{2}=\frac{k M m}{r}+\frac{N \hbar k M(\hat{\vec{\sigma}} \cdot \vec{r})}{2 c r^{3}}
$$

Hence

$$
\frac{\Omega_{1}^{2}}{\Omega_{2}^{2}}=\frac{r_{2}^{3}}{r_{1}^{3}}\left(1+\frac{N \hbar}{m c}\left(\frac{\left(\hat{\vec{\sigma}} \cdot \vec{r}_{1}\right)}{r_{1}^{2}}-\frac{\left(\hat{\vec{\sigma}} \cdot \vec{r}_{2}\right)}{r_{2}^{2}}\right)\right)
$$

where $(\hat{\vec{\sigma}} \cdot \vec{r})= \pm \frac{1}{2} r$. If the directions of $\hat{\vec{\sigma}}$ and $\vec{r}$ match to each other then $(\hat{\vec{\sigma}} \cdot \vec{r})=\frac{1}{2} r$. If the directions are opposite, then $(\hat{\vec{\sigma}} \cdot \vec{r})=-\frac{1}{2} r$. We estimate the correction to Kepler's third law, which follows from (66) for the satellite, in which every atom has an oriented spin (then $N / m=1 / m_{A}$ ), with $m_{A}$ standing by atomic mass. Let one oriented spin accounts for ten protons, then

$$
\frac{\hbar}{m_{A} c r}=10^{-23}
$$




\section{Conclusion}

The energy of (62), per one spin, is too small for modern physical effects. Even one mole of a substance gives the contribution of only one electron-volt. However research of the interaction of the matter spin and gravity would deepen our knowledge about the surrounding macrocosm, and perhaps a microcosm. The effects of torsion could play a role in the case of cosmic objects. Particularly, the disk structure of the space jet induces a torsion perpendicular to the plane of the jet, which might be a cause of observed polarization in the jets and jet emission currently assigned to the influence of the magnetic field jet.

There is another effect associated with this torsion. If you watch a satellite, moving by a parallelogram, then the parallelogram does not close and the defect is [11]:

$$
\Delta x^{\alpha}=\Gamma_{\beta \gamma}^{\alpha} S^{\beta \gamma}
$$

where $S^{\beta \gamma}$ is a pseudotensor of the parallelogram square. Since the pseudotensor of the square is antisymmetric by the indices and as it is clear from (69), the effect is entirely given by torsion, particularly by its traceless part. Taking the circle instead of the parallelogram, in the case of the torsion (48) we obtain

$$
\Delta x=\pi R^{2} \cdot \frac{r_{g}}{3 R^{2}} \approx r_{g} \approx 1 \mathrm{~cm}
$$

This value gives the angular dimesion which is observed from the Earth while watching to the satellite from the distance of $100 \mathrm{~km}$ :

$$
\Delta \varphi=\frac{\Delta x}{R}=\frac{1 \mathrm{~cm}}{100 \mathrm{~km}} \approx 10^{-7},
$$

which corresponds to the one thousandth of the second per one revolution.

\section{References}

[1] Dorofeev V. Yu. arxiv.org math-ph:0908.3247v1 (2009)

[2] Dorofeev V. Yu. arxiv.org gr-qc:1003.3228 (2010)

[3] Nesterov A. I. and Sabinin L. V. Phys. Rev. D62, 081501 (2000).

[4] Gogberashvili M. hep-th/0409173.

[5] Fock V., Ivanenko D., C. R., Paris 188, 1470 (1929).

[6] Eisenhart L. P. Riemannian gyometry. 1926.

[7] Sokolov A. Ivanenko D. Quantum theory field. 1952.

[8] Helzen F., Martin A. Quarks and leptons. 1987.

[9] Brill D. R., Wheeler J. A. Rev. Mod. Phys., 29, 465, (1957). 
[10] Okun L. B. Leptons and quarqs. 1990.

[11] Barvinsky A. O., Obuhov Yu. N., Ponomaryev V. N. Of the geometrydynamic methods and the gage approach to the theory of gravitational interactions.. 1985. 